\title{
A Novel Approach for Enhancing the Visual Perception of Ribs in Chest Radiography
}

\author{
Mira Park, Jesse S. Jin, Suhuai Luo \\ School of Design, Communication and Information Technology \\ The University of Newcastle \\ NSW 2308, Australia \\ Mira.park@newcastle.edu.au
}

\begin{abstract}
Intensity adjustment is an image enhancement technique that maps an image's intensity values to a new range. However, this intensity adjustment does not effectively enhance particular structures such as ribs on a $2 \mathrm{D}$ chest radiograph. In this paper, we develop a new method using a lopsided hemiellipsoid cavity to deflate lungs. This is necessary in order to enhance the unclear ribs resulting from air-filled lungs in a typical chest radiograph procedure.
\end{abstract}

\section{INTRODUCTION}

In recent years, digital medical imaging techniques have advanced rapidly, primarily from the advancements in the picture archiving and communication system (PACS). Medical imaging techniques are also proving to be powerful tools for patient diagnosis, treatment and surgery. One of the important functions of PACS is to display radiographs on screen. The availability of many high quality display unit as a part of PACS makes it possible for radiologists to investigate the images of their preference. For the conventional chest radiographs, a main function is to enhance a particular structure rather than display a 3D reconstruction in computer tomography or magnetic resonance imaging.

Image enhancement techniques are used to improve an image, where 'improve' is defined to make certain features easier to see by modifying the intensities [1]. Intensity adjustment is an image enhancement technique that maps an image's intensity values to a new range. If the new range is $[0$, 1], it maps a low value to 0 , and a high value to 1 . The values can be mapped linearly to values between 0 to 1 or can be mapped nonlinearly. However, this intensity adjustment is based on its intensity value, so it does not usefully enhance a particular structure such as ribs on 2D chest radiograph. The conventional $2 \mathrm{D}$ chest radiography is still the first exam for a sudden stomachache during sports. The cause of this symptom might be a broken bone or other organ abnormalities. However, the ribs are not clearly visible caused by the typical chest radiograph procedure.

The X-ray technologist will typically ask the patient to be still, to take a deep breath and to hold it. This not only reduces the possibility of a blurred image but also enhances the quality of the lung image since air-filled lungs are easier to see on X- ray film than deflated lungs. However, the air-filled lungs reduce the intensity of the ribs, especially in the inner part of the lung field. This means that ribs in the inner part of lung field have a lower density than the other parts close to the boundary of the lung field. Therefore, it is useful to make the intensity similar over the whole ribs, resulting clearer visibility of the ribs.

A high pass filter [5], a gradient operator [6], and Gaussian filter $[7,8,9]$ have been used for rib edge enhancement in preprocessing. However, none of these take into account the difference between the ribs at the inner part of the lung field and the boundary area of the lung field.

In this paper, we use the lopsided hemi-ellipsoid cavity to deflate lungs for enhancing the ribs on chest radiographs.

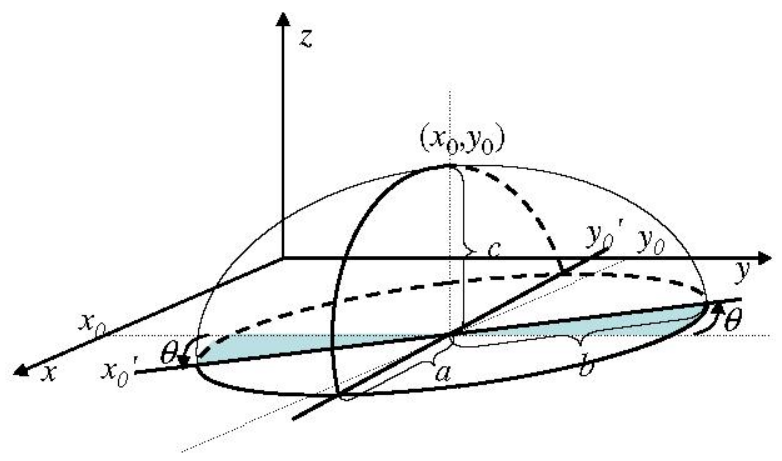

Fig. 1. The model of the lopsided hemi-elliptical cavity

\section{LOPSIDED HEMI-ELLIPSOID CAVITY}

The lopsided hemi-ellipsoid cavity is derived from our previous work [2]. The lung area's vertical center line is presented with a gradient. Therefore, the hemi-ellipsoid cavity with a right angle, presented in [2] does not precisely deflate the lung region. The lopsided hemi-ellipsoid cavity solved this problem by considering the characteristic of lopsided lung shape. 


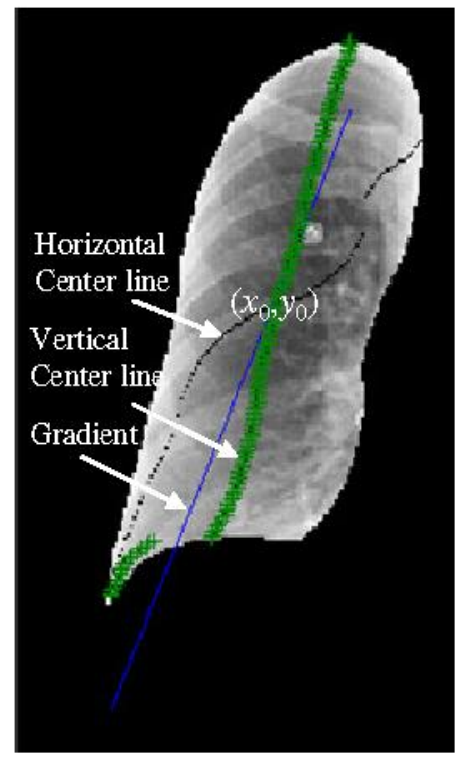

centre lines and gradient

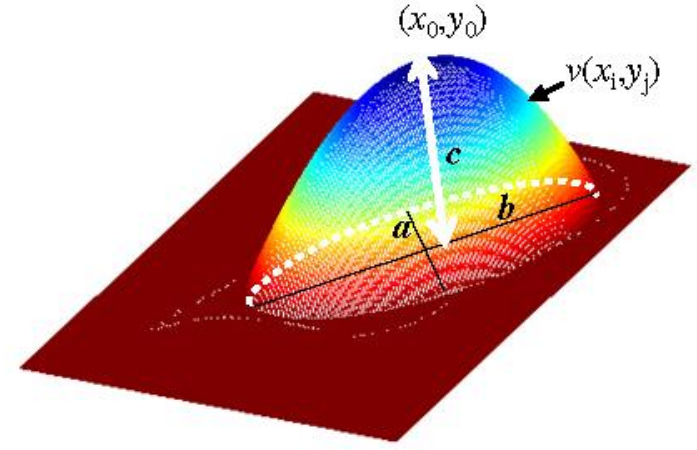

lopsided hemi-elliptical cavity, $v\left(x_{i}, y_{i}\right), 3 \mathrm{D}$ view

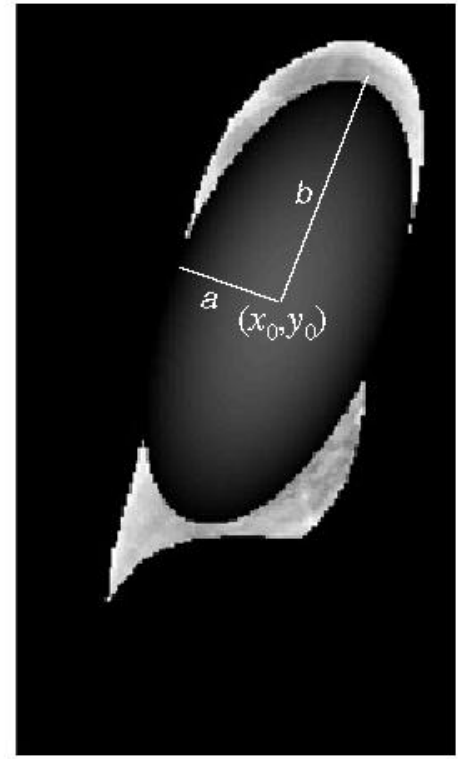

$2 \mathrm{D}$ view

Fig. 2. Lopsided hemi-elliptical cavity and its parameters

\section{A. Lopsided hemi-ellipsoid cavity}

As shown in Fig. 1, the center of the ellipsoid is $\left(x_{0}, y_{0}\right)$. We form the equation for the hemi-ellipsoid, $z$, where $z>=0$ as follows,

$$
z^{2}=c^{2} \times\left(1-\frac{\left(x-x_{0}\right)^{2}}{a^{2}}-\frac{\left(y-y_{0}\right)^{2}}{b^{2}}\right)
$$

$a, b, c$ and $\theta$ are constant values, which should be decided by the lung shape. The first component $a$ is the half length of the lung width, the second component $b$ is the half length of the lung height and the third component $c$ is the intensity value at $f\left(x_{0}, y_{0}\right)$ where $f$ is the input chest radiograph. We then use a linear regression of vertical center line of a lung region to obtain the gradient, $\theta$. The lopsided hemi-ellipsoid cavity, $v\left(x_{i}\right.$, $y_{i}$ ), is formulated as follows.

$$
\begin{aligned}
& v\left(x_{i}, y_{i}\right)= \\
& \sqrt{c^{2} \times\left(\begin{array}{l}
1-\frac{\left(\cos (\theta)\left(x_{i}-x_{0}\right)-\sin (\theta)\left(y_{i}-y_{0}\right)\right)^{2}}{a^{2}} \\
-\frac{\left(\sin (\theta)\left(x_{i}-x_{0}\right)+\cos (\theta)\left(y_{j}-y_{0}\right)\right)^{2}}{b^{2}}
\end{array}\right)}
\end{aligned}
$$

where $i$ is 1 to $n$ and $j$ is 1 to $m$ when the size of the input chest radiograph is $n \times m$ which includes only one side of lung regions (eg., right lung or left lung). We form a lopsided hemielliptical cavity with the values, $v\left(x_{i}, y_{i}\right)$, when they are greater than 0 or equal to 0 . Fig. 2 shows the example of a lopsided hemi-elliptical cavity on a chest radiograph.

\section{B. Enhancing the ribs}

We add a lopsided hemi-elliptical cavity from lung region to deflate the lung using the following condition: if $v\left(x_{i}, y_{j}\right) \geq 0$ then $f^{\prime}\left(x_{i}, y_{j}\right)=f\left(x_{i}, y_{j}\right)+v\left(x_{i}, y_{j}\right)$; elsef $f^{\prime}\left(x_{i}, y_{j}\right)=f\left(x_{i}, y_{i}\right)$.

The addition of the cavity means that there is a gradual increase of intensity from the boundary to the center of the lung region. Fig. 2 shows the two and three dimensions view of the lopsided hemi-elliptical cavity.

\section{PERFORMANCE ANALYSIS}

We tested 35 standard Posteroanterior (PA) 14" $\times 17$ " chest radiographs, selected in the Department of Radiology, St. Vincents Hospital. The radiographs were digitised with a Kodak Lumiscan Film Digitiser. The resolution of the digitised images is $0.175 \mathrm{~mm}$ with matrix size of 2048 by 2486 , and the images are subsampled to 256 by 310 to reduce the unnecessary points and edges that could be extracted. Our method applies to the segmented lung field using a lung field segmentation based on knowledge developed by Brown [3] and extended by Park [4].

We automatically detected the rib edges on both the inspiration and the expiration lungs using our previous rib edge detection method [2].

The true positives the false positives of the detected rib edges from both lungs are compared in the amount of pixels (see Fig. 3,4). 


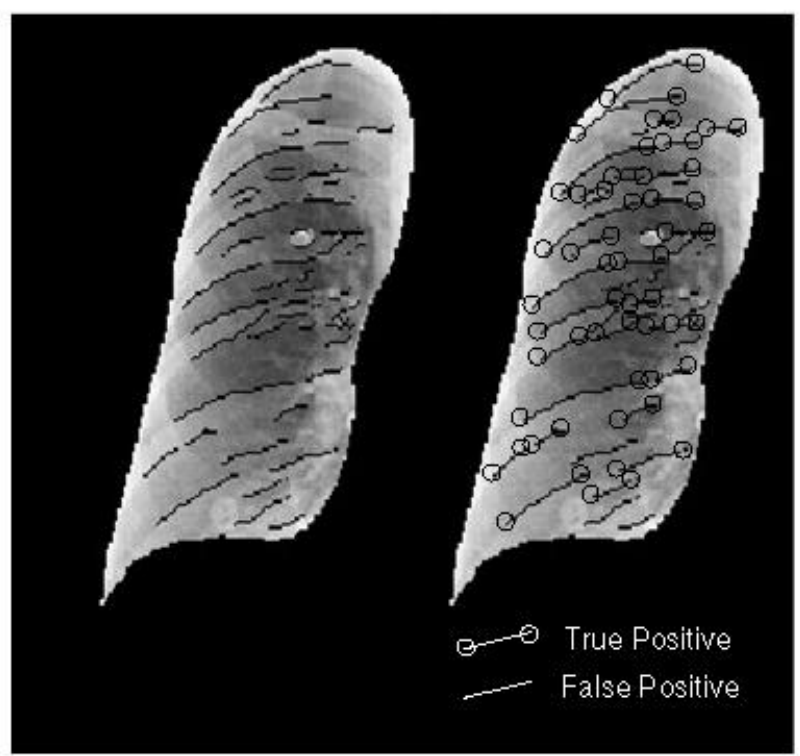

(a)

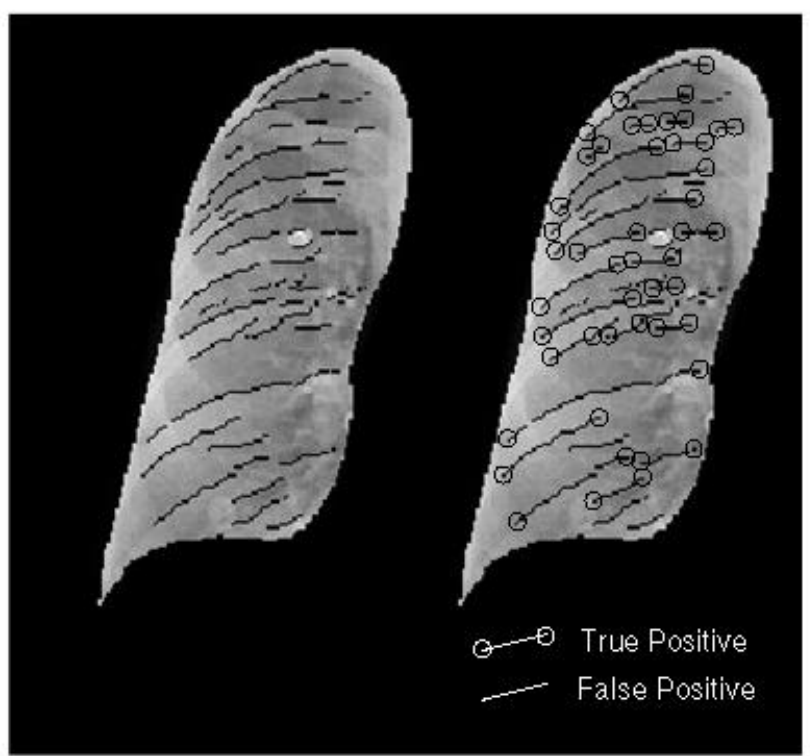

(b)

Fig. 3. The detected rib edge candidates on (a) inspiration (input) lung and (b) expiration lung

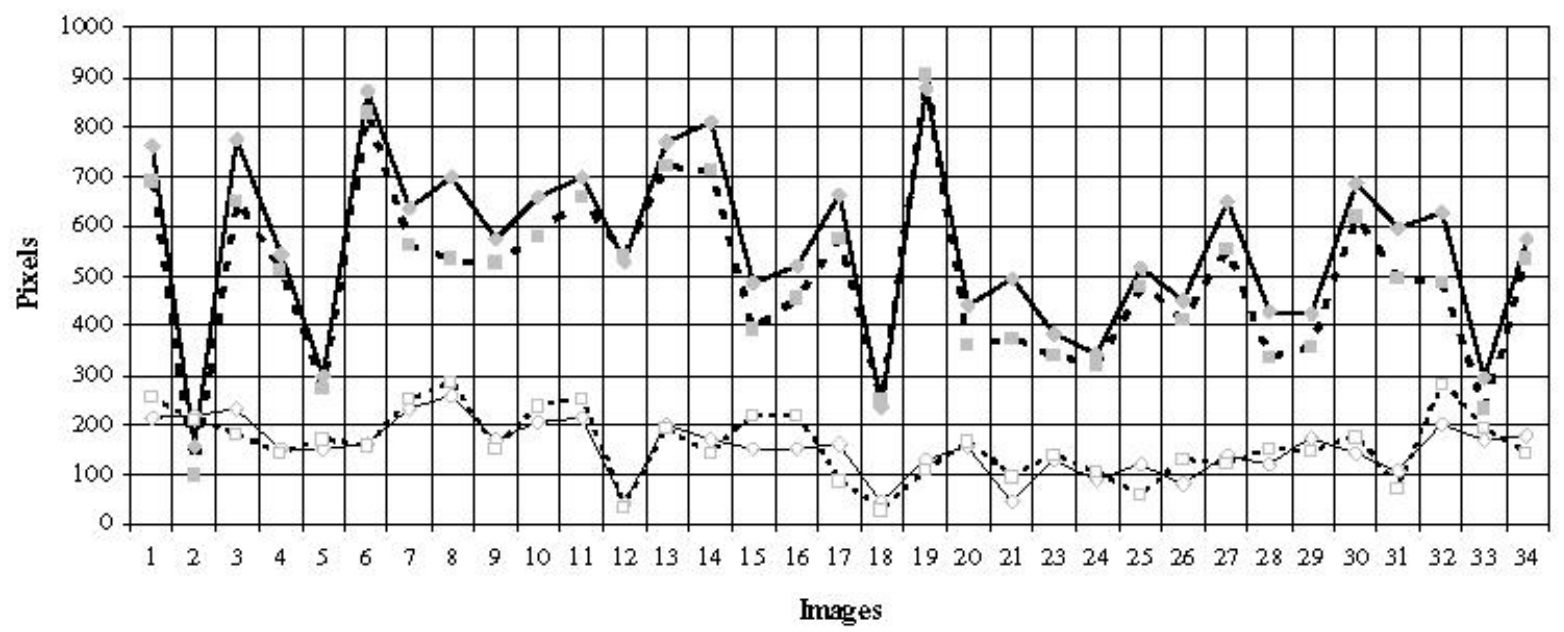

- Expiration(True Positive) - -- - Inspiration(True Positive) - - Expiration(False Positive) -... $\cdots$ Inspiration(False Positive)

Fig. 4. Plotting of the True Positive and False Positive rib edges on the inspiration and expiration lungs

Our technique produced average $15.1 \%$ more true positives of rib edges with $9.4 \%$ less false positives than the input (inspiration) lungs.

\section{TV. CONCL USION}

We presented a novel approach for enhancing the visual perception of ribs on chest radiography. The manifestations of many congenital and acquired conditions can be seen in the ribs of children [10]. When taking the X-ray, the X-ray technologist asks the patient to breathe out and hold it instead of taking a deep breath in order to clearly present the ribs. We can apply the method to produce the same effect on the X-ray taken by typical procedure of breathing in.

Our test image database consists of normal chest radiographs as well as abnormal chest radiographs which may include unclear presentation of the lung regions. As the result shows, our technique clearly improved the visual perception of the ribs on the chest radiographs which do not include serious texture abnormalities but reduced the visual perception of the 


\section{IEEE/ICME International Conference on Complex Medical Engineering}

ribs on the chest radiographs which include texture abnormalities. The texture abnormalities usually present as high intensity and our technique increases the intensity, so in this case the image quality will be reduced. Therefore, it will be effective to have this process as an option just like the intensity adjustment function in image processing tools and to manually adjust $a, b, c$, and $\theta$ for precise image display.

\section{REFERENCES}

[1] Gonzalez R.C. and Woods R.E., Digital Image Processing, AddisonWesley Publishing Company, 1993

[2] Park M., Jin J., and Wilson, L., "Detection and labeling ribs on expiration chest radiographs", The Physics of Medical Imaging conference at SPIE, 4(21): 1021-1031, San Diego USA, Feb 2003

[3] Brown M., Wilson L., Doust B., Gill R., and Sun C., "Knowledge-based Method for Segmentation and Analysis of Lung Boundaries in Chest Xray images", Computerized medical imagin and graphics, 22:463-
477, 1998

[4] Park M., Wilson L., and Jin J., "Automatic Extraction of Lung Boundaries by a Knowledge-based Method", Visual Information Processing, 2:14-19, 2001

[5] Wechsler H. and Skansky, "Automatic Detectin of Rib Contours in Chest Radiographs", International Joint Conference on Artificial Intelligence, 688-694, 1975

[6] Daponte S.J. and Fox D.M., "Enhancement of Chest Radiographs with Gradient Operators", IEEE Transactions on Medical Imaging, 7:109116,1988

[7] Sugahara T, Yanagihara Y. and Sugimoto N., "Construction of Structural Edge Map on Chest Radiograph using Hough Transformation and Line Connection", Systems and Computers in Japan, 26(6):71-78, 1995

[8] Vogelsang F., Weiler F., Lesch T., Wein B. and Gunther R.W., "Compensation of Rib Structures in Digital X-rays of the Chest For Diagnose Assistance", Computer Assisted Radiology, 1020, 1996

[9] Ginneken B. and Romeny B, "Automatic Delineation of Ribs in Frontal Chest Radiographs", Image Processing in SPIE, 3979:825-826, 2000

[10] Glass B.J.R, Norton I.K., Mitre A.S. and Kang E., "Pediatric Ribs: A Spectrum of Abnormalities", RadioGraphics, 22(1):87-104, 2002 